\title{
A CLASS OF REDUCIBLE SYSTEMS OF QUASI-LINEAR PARTIAL DIFFERENTIAL EQUATIONS*
}

\section{By CHONG-WEI CHU (Northrop Norair, Hawthorne, California)}

1. Introduction. Quasi-linear partial differential equations, occurring frequently in engineering problems, are often difficult to solve. This note presents a class of systems of quasi-linear equations reducible to a single linear heat equation, and gives an example of viscous fluid flow.

2. Reduction to a linear equation. The system of $n$ equations under consideration is of the form

$$
\frac{\partial u_{i}}{\partial t}+F_{i} \frac{\partial u_{i}}{\partial x_{j}}=G_{i} \frac{\partial u_{i}}{\partial x_{i}} \frac{\partial u_{i}}{\partial x_{j}}+k \frac{\partial^{2} u}{\partial x_{j} \partial x_{i}}+H_{i} R_{i}, \quad(i, j=1, \cdots, n)
$$

where summation convention is adopted with index $i$ not summed; $F_{i}, G_{i}$, and $H_{i}$ are functions of $u_{i}$ at least twice continuously differentiable; $k$ is a constant and $R_{i}$ a continuously differentiable function of $t, x_{1}, \cdots, x_{n}$. With some restrictions on $F_{i}, G_{i}$, $H_{i}$, and $R_{i}$, Eq. (1) can be reduced to a heat equation in $n$ dimensions.

Consider the transformation

$$
F_{i}\left(u_{i}\right)=-\frac{2 k}{\phi} \frac{\partial \phi}{\partial x_{i}}
$$

corresponding to which the following relations are true:

$$
\begin{gathered}
F_{i}^{\prime} \frac{\partial u_{i}}{\partial t}=\frac{2 k}{\phi^{2}} \frac{\partial \phi}{\partial t} \frac{\partial \phi}{\partial x_{i}}-\frac{2 k}{\phi} \frac{\partial^{2} \phi}{\partial t \partial x_{i}} \\
F_{i}^{\prime} \frac{\partial u_{i}}{\partial x_{i}}=\frac{2 k}{\phi^{2}} \frac{\partial \phi}{\partial x_{i}} \frac{\partial \phi}{\partial x_{i}}-\frac{2 k}{\phi} \frac{\partial^{2} \phi}{\partial x_{i} \partial x_{i}} \\
F_{i}^{\prime} \frac{\partial^{2} u_{i}}{\partial x_{i} \partial x_{i}}=-\frac{4 k}{\phi^{3}} \frac{\partial \phi}{\partial x_{i}} \frac{\partial \phi}{\partial x_{i}} \frac{\partial \phi}{\partial x_{i}}+\frac{2 k}{\phi^{2}} \frac{\partial^{2} \phi}{\partial x_{i} \partial x_{i}} \frac{\partial \phi}{\partial x_{i}}+\frac{4 k}{\phi^{2}} \frac{\partial \phi}{\partial x_{i}} \frac{\partial^{2} \phi}{\partial x_{i} \partial x_{i}} \\
-\frac{2 k}{\phi} \frac{\partial^{3} \phi}{\partial x_{i} \partial x_{i} \partial x_{i}}-\frac{4 k^{2}}{\phi^{2}} \frac{F_{i}^{\prime \prime}}{F_{i}^{\prime 2}}\left(\frac{1}{\phi} \frac{\partial \phi}{\partial x_{i}} \frac{\partial \phi}{\partial x_{i}}-\frac{\partial^{2} \phi}{\partial x_{i} \partial x_{i}}\right)\left(\frac{1}{\phi} \frac{\partial \phi}{\partial x_{i}} \frac{\partial \phi}{\partial x_{i}}-\frac{\partial^{2} \phi}{\partial x_{i} \partial x_{i}}\right),
\end{gathered}
$$

where prime denotes differentiation with respect to $u_{i}$ and index $i$ is not summed. Substitution of (3) into (1) yields, with $i$ not summed,

$$
\begin{aligned}
\frac{2 k}{\phi^{2}}\left(\frac{\partial \phi}{\partial x_{i}}-\phi \frac{\partial}{\partial x_{i}}\right) & \left(\frac{\partial \phi}{\partial t}-k \frac{\partial^{2} \phi}{\partial x_{i} \partial x_{i}}\right)=F_{i}^{\prime} H_{i} R_{i} \\
& +\frac{4 k^{2}}{\phi^{2} F_{i}^{\prime 2}}\left(G_{i} F_{i}^{\prime}-k F_{i}^{\prime \prime}\right)\left(\frac{1}{\phi} \frac{\partial \phi}{\partial x_{i}} \frac{\partial \phi}{\partial x_{i}}-\frac{\partial^{2} \phi}{\partial x_{i} \partial x_{i}}\right)\left(\frac{1}{\phi} \frac{\partial \phi}{\partial x_{i}} \frac{\partial \phi}{\partial x_{i}}-\frac{\partial^{2} \phi}{\partial x_{i} \partial x_{i}}\right) .
\end{aligned}
$$

Noting that the left-hand side of (4) is the partial derivative of a function with respect to $x_{i}$, we set

$$
G_{i} F_{i}^{\prime}-k F_{i}^{\prime \prime}=0, \quad F_{i}^{\prime} H_{i}=1, \quad R_{i}=-\partial P / \partial x_{i},
$$

*Received August 7, 1964. 
where $i$ is not summed, and rewrite (4) as

$$
\frac{\partial}{\partial x_{i}}\left[\frac{2 k}{\phi}\left(\frac{\partial \phi}{\partial t}-k \frac{\partial^{2} \phi}{\partial x_{i} \partial x_{i}}\right)-P\right]=0,
$$

which can be integrated to give

$$
\frac{\partial \phi}{\partial t}=k \frac{\partial^{2} \phi}{\partial x_{i} \partial x_{i}}+\left[c(t)+\frac{P}{2 k}\right] \phi,
$$

a linear heat equation with linear heat generation. We have shown that solutions of a system of $n$ quasi-linear equations (1) can be obtained from the solutions of a linear equation (6). It is noted, however, that (6) will not yield all solutions of (1) because of the limitation imposed on $F_{i}$ by the transformation(2).

Thus, the system of equations (1) can be reduced to a single linear equation whenever (5), which may be termed the "reducibility conditions," is satisfied. A necessary and sufficient condition for the first two of (5) is that $F_{i}, G_{i}$, and $H_{i}$ are derived from a generating function $f_{i}\left(u_{i}\right)$ by the formulae

$$
\begin{aligned}
& F_{i}=\int^{u_{i}} f_{i}(u) d u, \\
& G_{i}=k d\left(\ln f_{i}\right) / d u_{i}, \\
& H_{i}=f_{i}^{-1},
\end{aligned}
$$

where $i$ is not summed. A necessary and sufficient condition for the last of (5) is that the Strokes tensor $\mathrm{S}$ for $R_{i}$ vanishes identically

$$
S_{i j}=\frac{\partial R_{i}}{\partial x_{i}}-\frac{\partial R_{i}}{\partial x_{i}}=0 .
$$

3. Navier-Stokes equations. As an example, let us consider the Navier-Stokes equations for incompressible fluid flow

$$
\frac{\partial u_{i}}{\partial t}+u_{i} \frac{\partial u_{i}}{\partial x_{i}}=-\frac{1}{\rho} \frac{\partial p}{\partial x_{i}}+\nu \frac{\partial^{2} u_{i}}{\partial x_{i} \partial x_{i}}, \quad i, j=1,2,3,
$$

where $u_{i}$ is the velocity component in the $x_{i}$ direction, $p$ the pressure, $\rho$ the density, and $\nu$ the kinematic viscosity, which is assumed constant. It is easily checked that the reducibility conditions (5) are satisfied; hence through the transformation

$$
u_{i}=-\frac{2 \nu}{\theta} \frac{\partial \theta}{\partial x_{i}},
$$

Eq. (9) reduces to a linear heat equation

$$
\frac{\partial \theta}{\partial t}=\nu \frac{\partial^{2} \theta}{\partial x_{i} \partial x_{i}}+\frac{p\left(t, x_{1}, x_{2}, x_{3}\right)}{2 \rho \nu} \theta .
$$

It should be noted that the Navier-Stokes equation with no pressure gradient was reduced to the heat conduction equation by Cole [1]. The one-dimensional case without the pressure term was studied by Burgers [2] and Cole [1]. 
It is permissible to view (11) as describing a mathematical model of some viscous flow and to solve (11) as an initial value problem in infinite space with prescribed pressure $p\left(t_{1}, x_{1}, x_{2}, x_{3}\right)$; then the velocity field so obtained will need a corresponding source distribution as given by

$$
Q\left(t, x_{1}, x_{2}, x_{3}\right)=-2 \nu \frac{\partial^{2} \ln \theta}{\partial x_{i} \partial x_{i}}
$$

to satisfy conservation of mass. On the other hand, when the source distribution is specified (this case being more physical), for instance $Q \equiv 0$, Eq. (11) may be transformed into a Bernoulli's equation through elimination of $\nabla^{2} \theta$. This result is not surprising since in combination with the continuity equation the viscous term in (9) becomes $\nu \nabla \times(\nabla \times \mathfrak{u})$ that drops off under the assumption of irrotationality implied by (10). Conversely, the nonlinear Bernoulli's equation for inviscid flow

$$
\frac{\partial \phi}{\partial t}+\frac{1}{2} \frac{\partial \phi}{\partial x_{i}} \frac{\partial \phi}{\partial x_{i}}+\frac{p}{\rho}=0
$$

may be converted into a linear heat equation similar to (11) by means of the equation of continuity and a change of variable $\phi=\ln \theta$.

4. Some reducible equations. A few simple forms of (1) will be listed for reference. For simplicity of presentation, only one-dimensional equations are given. Corresponding to the generating functions $f=0,1, e^{u}, n u^{n-1}, \ln u,-\sin u$, and $\cos u$ in (7), the following equations belong to the reducible class (1):

$$
\begin{gathered}
\frac{\partial u}{\partial t}=k \frac{\partial^{2} u}{\partial x^{2}}, \\
\frac{\partial u}{\partial t}+u \frac{\partial u}{\partial x}=k \frac{\partial^{2} u}{\partial x^{2}}+R(t, x), \\
\frac{\partial u}{\partial t}+e^{u} \frac{\partial u}{\partial x}=k\left(\frac{\partial u}{\partial x}\right)^{2}+k \frac{\partial^{2} u}{\partial x^{2}}+e^{-u} R(t, x), \\
\frac{\partial u}{\partial t}+u^{n} \frac{\partial u}{\partial x}=\frac{k n(n-1)}{u}\left(\frac{\partial u}{\partial x}\right)^{2}+k \frac{\partial^{2} u}{\partial x^{2}}+\frac{R(t, x)}{n u^{n-1},} \\
\frac{\partial u}{\partial t}+u(\ln u-1) \frac{\partial u}{\partial x}=\frac{k}{u \ln u}\left(\frac{\partial u}{\partial x}\right)^{2}+k \frac{\partial^{2} u}{\partial x^{2}}+\frac{R(t, x)}{\ln u}, \\
\frac{\partial u}{\partial t}+\left(\begin{array}{l}
\cos u \\
\sin u
\end{array}\right) \frac{\partial u}{\partial x}=-k\left(\begin{array}{c}
\cot u \\
\tan u
\end{array}\right)\left(\frac{\partial u}{\partial x}\right)^{2}+k \frac{\partial^{2} u}{\partial x^{2}}+\left(\begin{array}{c}
-\csc u \\
\sec u
\end{array}\right) R(t, x),
\end{gathered}
$$

where $R(t, x)$ is any function integrable with respect to $x$. It may be noted that in the one-dimensional case transformation (2) imposes no restriction on $u$ more than the requirement for existence of solutions; hence every solution of the original equation may be obtained from the corresponding heat equation. Likewise, the $n$-dimensional systems may be derived from $n$ generating functions, some or all of which may be identical. The one-dimensional equations listed above may provide a good visualization of the $n$-dimensional systems. 


\section{REFERENCES}

[1] J. D. Cole, On a Quasi-Linear Parabolic Equation Occurring in Aerodynamics, Quart. Appl. Math. 9, 225-236 (1951)

[2] J. M. Burgers, A Mathematical Model Illustrating the Theory of Turbulence, Adv. Appl. Mech., Vol. I, Academic Press, New York, 1948, p. 171 